\title{
Probing nucleon's spin structures with polarized Drell-Yan in the Fermilab SpinQuest experiment
}

Andrew Chen ${ }^{* 1}$, J.C. Peng ${ }^{1}$, C. H. Leung ${ }^{1}$, M. Tian ${ }^{1}$, N. Makins ${ }^{1}$, M. Brooks ${ }^{2}$, A. Klein ${ }^{2}$, D. Kleinjan ${ }^{2}$, K. Liu²(CoSpokesperson), M. McCumber ${ }^{2}$, P. McGaughey ${ }^{2}$, J. Miraal-Martinez ${ }^{2}$, C. Da Silva ${ }^{2}$, Sho Uemura ${ }^{2}$, M. Jen $^{2}$, X. Li ${ }^{2}$, J. Arrington ${ }^{3}$, D. Geesaman ${ }^{3}$, P. E. Reimer ${ }^{3}$, C. Brown ${ }^{4}$, R. J. Tesarek ${ }^{4}$, S. Sawada ${ }^{5}$, W. Lorenzon ${ }^{6}$, R. Raymond ${ }^{6}$, K. Slifer ${ }^{7}$, D. Ruth ${ }^{7}$, Y. Goto ${ }^{8}$, K. Nakano ${ }^{9}$, T.-A. Shibata ${ }^{9}$, D. Crabb ${ }^{10}$, D. Day ${ }^{10}$, D. Keller ${ }^{10}$ (CoSpokesperson), O. Rondon ${ }^{10}$, Z. Akbar ${ }^{10}$, J. Dunne ${ }^{11}$, D. Dutta ${ }^{11}$, L. El Fassi ${ }^{11}$, H. Jiang ${ }^{11}$, E. Kinney ${ }^{12}$, N, Doshita ${ }^{13}$, T. Iwata ${ }^{13}$, Y. Miyachi ${ }^{13}$, M. Daugherity ${ }^{14}$, D. Isenhower ${ }^{14}$, R. Towell ${ }^{14}$, S. Watson ${ }^{14}$, N. Rowlands ${ }^{14}$, Y. Ngenzi ${ }^{14}$, S. Pate ${ }^{15}$, V. Papavassiliou ${ }^{15}$, H. Yu ${ }^{15}$, F. Hossain ${ }^{15}$

${ }^{1}$ University of Illinois at Urbana-Champaign, Champaign, IL 61801;

${ }^{2}$ Los Alamos National Laboratory, Los Alamos, NM 87545;

${ }^{3}$ Argonne National Laboratory, Lemont, IL 60439;

${ }^{4}$ Fermi National Accelerator Laboratory, Batavia, IL 60510;

${ }^{5}$ KEK, Tsukuba, Ibaraki 305-0801, Japan;

${ }^{6}$ University of Michigan, Ann Arbor, MI 48109-1040;

${ }^{7}$ University of New Hampshire, Durham, NH 03824;

${ }^{8}$ RIKEN, Wako, Saitama 351-0198, Japan;

${ }^{9}$ Tokyo Institute of Technology, Meguro, Tokyo 152-8550, Japan;

${ }^{10}$ University of Virginia, Charlottesville, VA 22904;

${ }^{11}$ Mississippi State University, Starkville, MS 39762;

${ }^{12}$ University of Colorado, Boulder, CO 80309;

${ }^{13}$ Yamagata University, Yamagata, Yamagata 990-8560, Japan;

${ }^{14}$ Abilene Christian University, Abilene, TX 79601;

${ }^{15}$ New Mexico State University, Las Cruces, NM 88003

Although the proton was discovered about 100 years ago, its spin structure still remains a mystery. Recent studies suggest that the orbital angular momentum of sea quarks could significantly contribute to the proton's spin. The SeaQuest experiment, which recently completed data collection, probed the unpolarized light quark sea distributions of the proton using the Drell-Yan process. Its successor, the SpinQuest (E1039), will access the $\bar{u}$ and $\bar{d}$ Sivers functions using polarized $\mathrm{NH}_{3}$ and $\mathrm{ND}_{3}$ targets. A non-zero Sivers asymmetry, observed in SpinQuest, would be a strong indication of non-zero sea-quark orbital angular momentum. The SpinQuest experiment can also probe the sea quark's transversity distribution, which is relevant for the determination of proton's tensor charge. Recent study suggests that sea-quarks might contribute significantly to deuteron's tensor polarized structure functions. This can be further probed in SpinQuest using tensor polarized $\mathrm{ND}_{3}$ target. The current status and future plan of the experiment are presented.

23rd International Spin Physics Symposium - SPIN2018 -

10-14 September, 2018

Ferrara, Italy

* Speaker. 


\section{Introduction}

There has been intense theoretical and experimental interest in the subject of transverse momentum dependent (TMD) parton distributions of the nucleons [1] during the last two decades. For many years after the discovery of the partonic structure of the nucleons in the deep inelastic scattering (DIS), only their unpolarized and helicity distributions were investigated. New insights on the nucleon structure and QCD are expected to be revealed by the novel TMD distributions. One example is the striking prediction [2] of the process dependence of certain TMDs, which remains to be tested conclusively with ongoing and future experiments. The recent advance of the lattice QCD, including the prospect of calculating the Bjorken- $x$ dependence of the parton distributions and TMDs [3], could also lead to a direct comparison between the experimental data and lattice calculations.

From the three transverse quantities of the nucleon and quark, namely, the nucleon's transverse spin, quark's transverse spin, and the quark's transverse momentum, three different correlations corresponding to three different TMDs can be formed. The Sivers function results from the correlation between quark's transverse momentum and nucleon's transverse spin [ [7]. The correlation between quark's and nucleon's transverse spins leads to the "transversity" distribution [1]. The correlation between quark's transverse spin and quark's transverse momentum gives the Boer-Mulders function [5]. Among the various types of TMDs, the bulk of recent interest and progress centered on these three TMDs.

The novel TMDs are accessible via experiments using either lepton or hadron beams. Most of the experimental information on the TMDs thus far has been collected from the semi-inclusive DIS (SIDIS) experiments carried out with muon or electron beams at DESY (HERMES), CERN (COMPASS), and the Jefferson Lab. Another important experimental tool to access the TMDs using hadron beams is the Drell-Yan process, which involves quark-antiquark annihilating into a virtual photon via electromagnetic interaction. As such, the Drell-Yan cross sections with unpolarized or transversely polarized nucleons are potentially sensitive to various TMDs of the colliding hadrons. First results on the Boer-Mulders functions have been extracted from the unpolarized Drell-Yan experiments with pion beams [6, 7] and proton beam [8, 9]. Very recently, Sivers function was probed in W-boson production (a generalized Drell-Yan process) using transversely polarized proton beam at RHIC by the STAR Collaboration [10] as well as in pion-induced Drell-Yan on a transversely polarized proton target at CERN by the COMPASS Collaboration [11].

In this paper, we present the physics motivation and experimental status of a recently approved polarized Drell-Yan experiment, SpinQuest (E1039), at Fermilab [12]. The SpinQuest experiment is a follow-up of the recently completed SeaQuest experiment [13], with an important new feature that transversely polarized targets will be implemented. The main physics goal of the SpinQuest is to measure proton's sea-quark Sivers function, which is expected to provide an unique information complementary to that obtained from the polarized Drell-Yan experiments at COMPASS and STAR. Additional physics opportunities of the SpinQuest, including the measurements of the nucleon's sea-quark transversity distribution and the tensor polarized structure function, $b_{1}$, of the deuteron, will also be discussed. 


\section{Probing Sea-quark Sivers Functions}

Sivers first suggested that the correlation between the transverse spin of the nucleon and the transverse momentum of the quark (antiquark) can lead to single-spin asymmetry in various processes [ [П]. The Sivers function is a time-reversal odd object. Its existence depends on initial or final state interactions via soft gluons [2]. As shown in Refs. [2, 14], such interactions are incorporated naturally by the gauge link required for a gauge-invariant definition of the TMD. The Sivers function is related to the forward scattering amplitude where the helicity of the nucleon spin is flipped. This helicity flip must involve the orbital angular momentum of the quark, hence the existence of the Sivers function indicates non-zero orbital angular momentum carried by the quark.

Measurements of the Sivers functions via the polarized SIDIS process with transversely polarized targets have been performed at HERMES [14, 15], COMPASS [16, 17, 18] and JLab [19]. Both valence and sea quark Sivers functions were extracted via global fits [20, 21] to existing data. The result confirmed the theoretical expectation of opposite sign for the $u$ and $d$ valence quark Sivers functions. Non-zero values, albeit with large uncertainties, were also obtained for the sea-quark Sivers functions [20, 21].

The connection between the Sivers function and the orbital angular momentum suggests that the Sivers function for antiquarks could be sizable. First of all, the meson-cloud model, which describes well [22] the observed $\bar{d} / \bar{u}$ asymmetry [23], implies that a significant fraction of nucleon's spin resides in the orbiting meson. Since mesons contain valence antiquarks, it is natural to expect that non-zero orbital angular momentum is carried by antiquarks. Secondly, using the chiral-quark soliton model, it was shown that antiquarks have a dominant contribution to proton's orbital angular momentum [24]. Finally, recent lattice calculation [25] also found a significant fraction of proton's spin originating from the $\bar{u}$ and $\bar{d}$ orbital angular momentum. All these theoretical results suggest that the sea-quark Sivers functions should be sizable and measurable. Proton-induced Drell-Yan experiment with transversely polarized target, such as the Fermilab SpinQuest experiment, provides a clean probe to extract sea-quark Sivers functions. Unlike the polarized SIDIS, where the effects of the sea-quark Sivers functions are often overshadowed by that of the valencequark Sivers functions, the polarized Drell-Yan with proton beam is predominantly sensitive to the sea-quark Sivers functions.

To measure the sea-quark Sivers functions, SpinQuest will use $120 \mathrm{GeV}$ proton beam bombarding on transversely polarized $\mathrm{NH}_{3}$ or $\mathrm{ND}_{3}$ targets. Using the upgraded SeaQuest dimuon spectrometer, the left-right azimuthal asymmetry, $A_{N}$, of dimuons due to the sea-quark Sivers functions in the target nucleon will be measured. This asymmetry is expressed as

$$
A_{N} \propto \frac{\sum_{q} e_{q}^{2}\left[f_{1}^{q}\left(x_{1}\right) \cdot f_{1 T}^{\perp, \bar{q}}\left(x_{2}\right)+1 \leftrightarrow 2\right]}{\sum_{q} e_{q}^{2}\left[f_{1}^{q}\left(x_{1}\right) \cdot f_{1}^{\bar{q}}\left(x_{2}\right)+1 \leftrightarrow 2\right]},
$$

where $f_{1}^{q}$ and $f_{1 T}^{\perp}$ denotes the unpolarized PDF and the Sivers function, respectively. The second term in both the numerator and denominator has negligible contribution due to the kinematic acceptance of the SpinQuest spectrometer. Figure 1 shows the projected sensitivities for the left-right asymmetry $A_{N}$ as a function of $x$ for the measurement on $\mathrm{NH}_{3}$ and $\mathrm{ND}_{3}$ targets. The green and yellow bands correspond to the calcuation of $A_{N}$ for the $\mathrm{NH}_{3}$ target using the Sivers functions obtained from two global fits to the SIDIS data [20, 21]. 


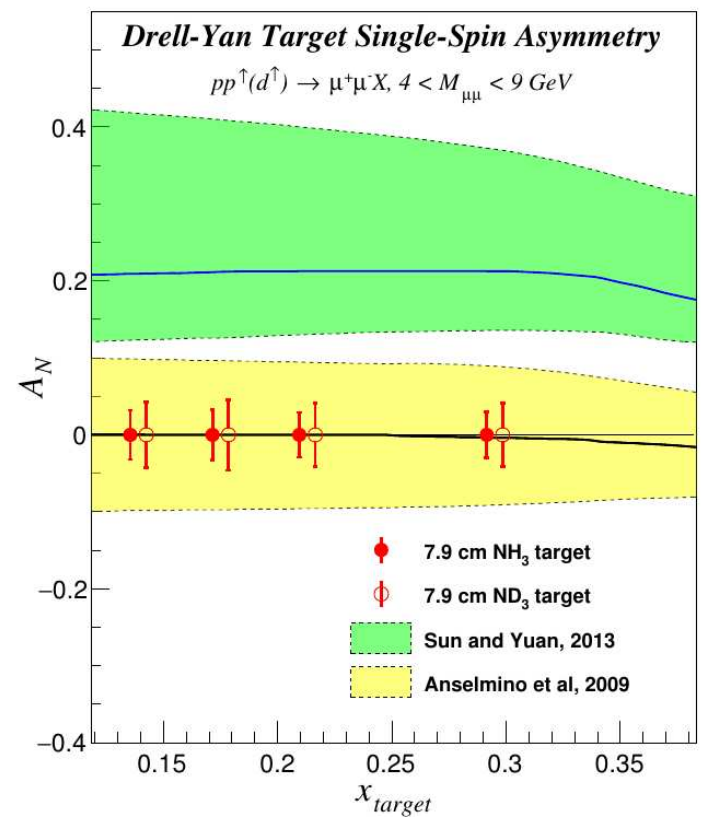

Figure 1: Projected sensitivities of the SpinQuest for the $A_{N}$ measurements on $\mathrm{NH}_{3}$ target. The two bands are calculations using the Sivers functions obtained from two different global fits to the SIDIS data [20, 21].

Figure 11 shows that the projected sensitivities of SpinQuest would allow a significant first measurement of the sea-quark Sivers function in the Drell-Yan process. A comparison between the $\mathrm{NH}_{3}$ and $\mathrm{ND}_{3}$ results could also be sensitive to the flavor dependence of the sea-quark Sivers function. If the magnitude of the sea-quark Sivers function is sizable, the sign of the sea-quark Sivers function might also be determined. The SpinQuest measurement would complement very well the COMPASS [11] and STAR [10] measurements, which are sensitive to the sign of the valence-quark Sivers functions.

\section{Probing the Sea-quark Transversity}

In addition to the primary physics goal of SpinQuest to measure the sea-qaurk Sivers function, an interesting potential by-product of SpinQuest is the measurement of nucleon's sea-quark transversity distribution. The transversity distribution describes the correlation between quark's transverse spin and nucleon's transverse spin. As a chiral-odd object, the transversity is not accesible in inclusive DIS, but can be extracted in semi-inclusive DIS or polarized Drell-Yan experiments, such as SpinQuest [1]. In order to probe the chiral-odd transversity distribution, another chiral-odd object is involved in the measurement. For the SIDIS, the chiral-odd Collins fragmentation function [26] or the interference fragmentation function are involved. For the Drell-Yan process, a convolution of a transversity distribution with either another transversity distribution or the chiral-odd Boer-Mulders function is involved.

Extensive efforts to measure the transversity distributions via SIDIS have been performed at HERMES [14, 27], COMPASS [28, 29], and JLab [19]. A global fit [30] to these SIDIS data, 
as well as the $e^{+} e^{-}$data from Belle [31] relevant for the Collins fragmentation function, has led to the extraction of the quark transversity distributions in the proton. However, in this global analysis, the sea-quark transversity distributions were assumed to be zero. Again, this reflects the challenge encountered in the SIDIS to separate the antiquark contribution from the dominant quark contribution.

The tensor charge of the proton is defined as the first moment of the difference of the quark and antiquark transversity distribution as

$$
\delta q=\int_{0}^{1}\left[h_{1}^{q}(x)-h_{1}^{\bar{q}}(x)\right] d x
$$

From the global fit to the SIDIS data, the $u$ and $d$ quark tensor charges were obtained [30] as shown in Figure 2 . While the tensor charge for the $d$ quark is in reasonable agreement with model calculations, Figure 目 shows a significant discrepancy between the data and model calculations (and lattice calculations) for the tensor charge of the $u$ quark. A possible explanation is that the sea-quark transversity distributions, which were neglected in the global analysis, might be quite sizable. It would be of great interest to measure the $\bar{u}$ and $\bar{d}$ transversity distributions using the Drell-Yan process.

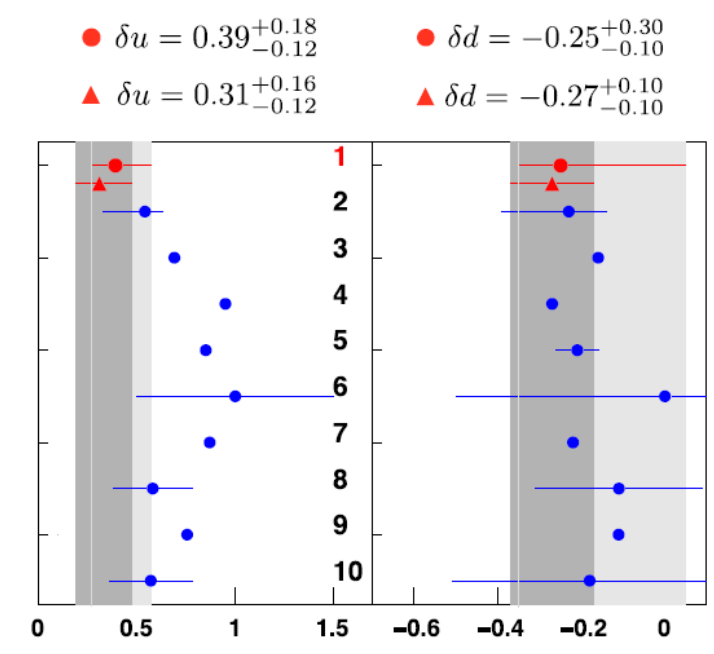

Figure 2: Tensor charges for the $u$ and $d$ quarks extracted in the global fit to SIDIS data [30]. Predictions from various models and the lattice calculations are also shown.

In the SpinQuest experiment, the antiquark transversity distributions can be accessed through the measurement of the correlation between the azimuthal angle of the charged leptons from the Drell-Yan process and the transverse polarization direction of the target nucleon. Specifically, the Drell-Yan differential cross section on a transversely polarized nucleon contains a term proportional to $\sin \left(2 \phi-\phi_{s}\right)$, where $\phi$ is the azimuthal angle of charged lepton in the dilepton rest frame and $\phi_{s}$ is the azimuthal angle of the nucleon's spin direction [11]. The amplitude of this term is proportional to the convolution of the quark Boer-Mulders function of the proton and the antiquark transversity distribution of the target proton. As the Boer-Mulders function can be determined from the $\cos 2 \phi$ distribution of the unpolarized Drell-Yan data [6, 7, 8, 9], it is quite feasible to probe the sea- 
quark transversity distributions. Further study of the expected sensitivity in SpinQuest for such measurement is underway.

\section{Study of Deuteron Tensor Polarization Function}

With tensor polarized $\mathrm{ND}_{3}$ target, the SpinQuest experiment can measure the tensor polarization function of the deuteron. Deuteron has spin 1 thus has three magnetic sub-states, $\mathrm{m}=+1,0$, -1. This leads to new PDF functions, $b_{1}, b_{2}, b_{3}$ and $b_{4}$. Among which $b_{1}$ is related to the difference between $|\mathrm{m}|=1$ and 0 . Figure 3 shows the HERMES SIDIS measurement [32] of $x b_{1}$ vs $x$, where $x$ is the Bjorken- $x$. The HERMES $b_{1}$ data suggest an oscillating pattern with a node occuring at $x \sim 0.3$, in qualitative agreement with a conventional description for $b_{1}$ [33]. The two curves in Figure 3 correspond to theoretical calculations [34] for the $x b_{1}$ distribution. The solid curve, which assumes non-zero antiquark contribution to $b_{1}$, gives significantly better description than the calculation (dashed curve) which assumes no antiquark $b_{1}$ contribution. This suggests a sizable antiquark contribution to the deuteron's $b_{1}$ structure function.

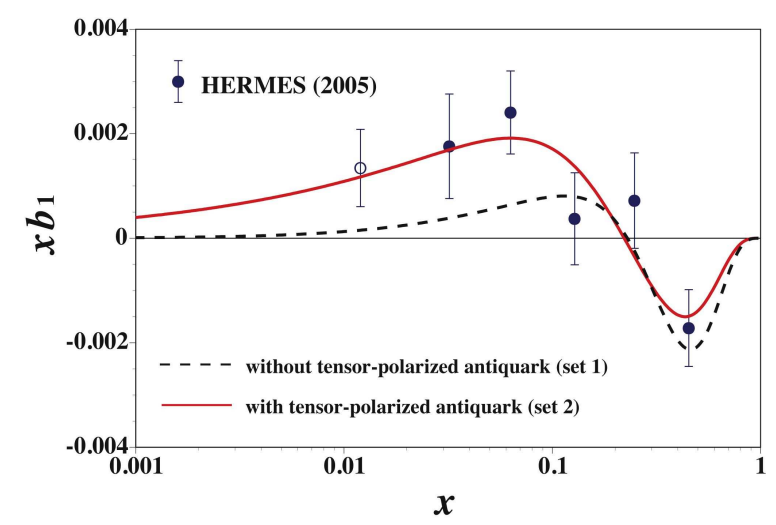

Figure 3: Comparison between the HERMES data [32] and the theoretical prediction [34]. The solid and dashed curves correspond to calculation with, or without, finite antiquark tensor polarization respectively.

The SpinQuest experiment can provide a direct measurement of the sea-quark contribution to the $b_{1}$ structure function. Using a tensor polarized $\mathrm{ND}_{3}$ target, the tensor polarization asymmetry, $A_{Q}$, in the Drell-Yan process can be measured. $A_{Q}$ is defined as the ratio of the unpolarized PDFs and the tensor-polarized PDFs [34]. Figure 4 shows the prediction for $A_{Q}$ for the SpinQuest kinematics [34]. The solid lines are the prediction assuming non-zero antiquark tensor polarization PDFs with various values of $x_{1}$, the momentum fraction of quark inside the beam proton. While the dashed lines assume zero antiquark tensor polarization. This shows that $A_{Q}$ can distinguish the two predictions, especially at the $x_{2}<0.2$ region. Figure 5 shows that the SpinQuest experiment covers the appropriate ranges of $x_{1}$ and $x_{2}$ to measure the $b_{1}$ of deuteron. Dedicated beam time to take both tensor polarized and unpolarized data would be necessary to make a quality measurement. 


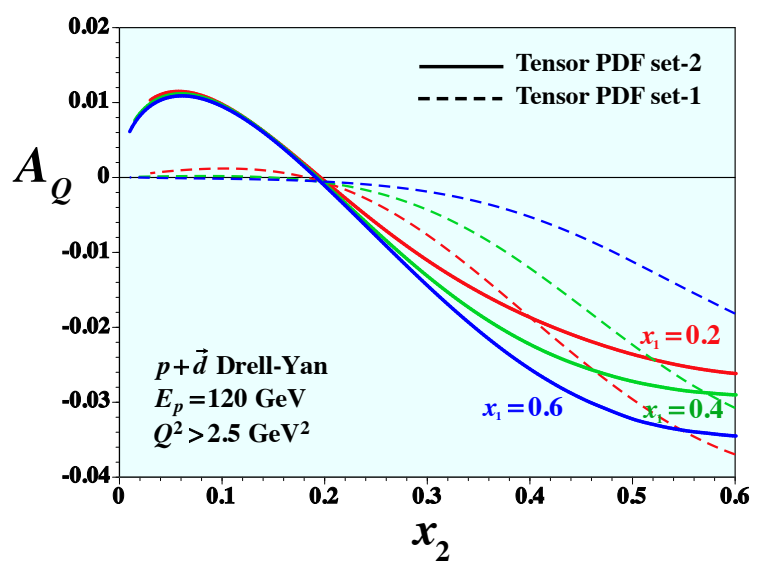

Figure 4: A prediction of the asymmetry $A_{Q}$ for the SpinQuest experiment based on two tensor PDF sets obtained from a fit to the HERMES data. [34] The solid curves were obtained with finite antiquark tensor polarization, while the dashed curves correspond to calculations assuming vanishing antiquark tensor polarization.

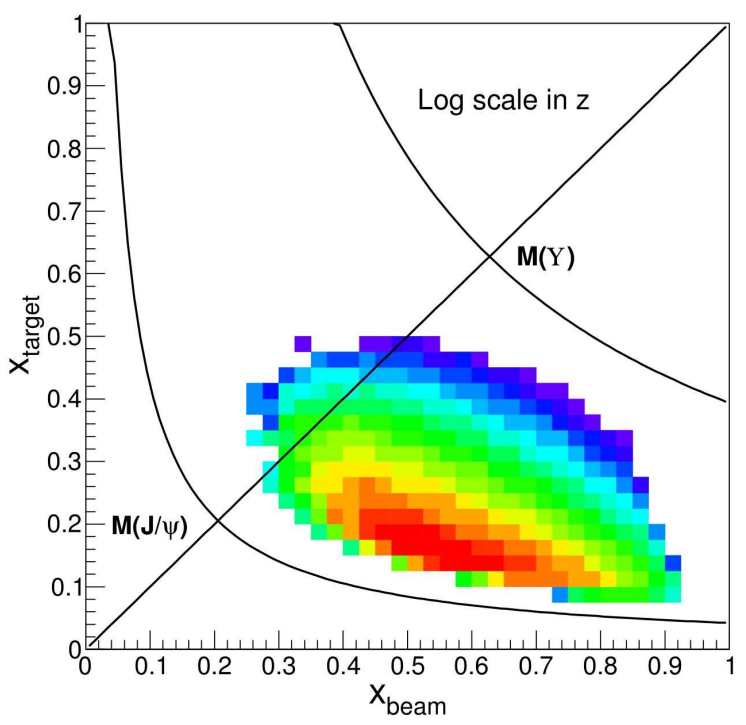

Figure 5: The 2D distribution of $x_{\text {target }}\left(x_{2}\right)$ vs $x_{\text {beam }}\left(x_{1}\right)$ showing that the SpinQuest can cover $x_{2}$ down to 0.1 to measure the tensor polarization function.

\section{The SpinQuest Experiment}

The SpinQuest spectrometer is basically the same as that used in the SeaQuest/E906 experiment [13], shown in Figure 6. The liquid $\mathrm{H}_{2}$ and $\mathrm{D}_{2}$ targets, used in the SeaQuest experiment, will be replaced by the polarized $\mathrm{NH}_{3}$ or $\mathrm{ND}_{3}$ target; also moved upstream by 1.5 meter to improve the separation of dimuon events from target and from dump. New fiber scintillators will be added in stations 1 and 2. This provides better $y$ position resolution thus improving the $z$ resolution and background rejection. The target is followed by a solid iron bending magnet, which also servers as 
a beam dump. Dimuons are tracked by stations 1-3 consisting of drift chambers and hodoscopes, while a second magnet (KMAG) is for analyzing the muon momentum. Station 4, placed downstream of an iron block, consists of hodoscope and proportional tubes for muon identification. The hit patterns of the hodoscopes are utilized to select muon events at the trigger level.

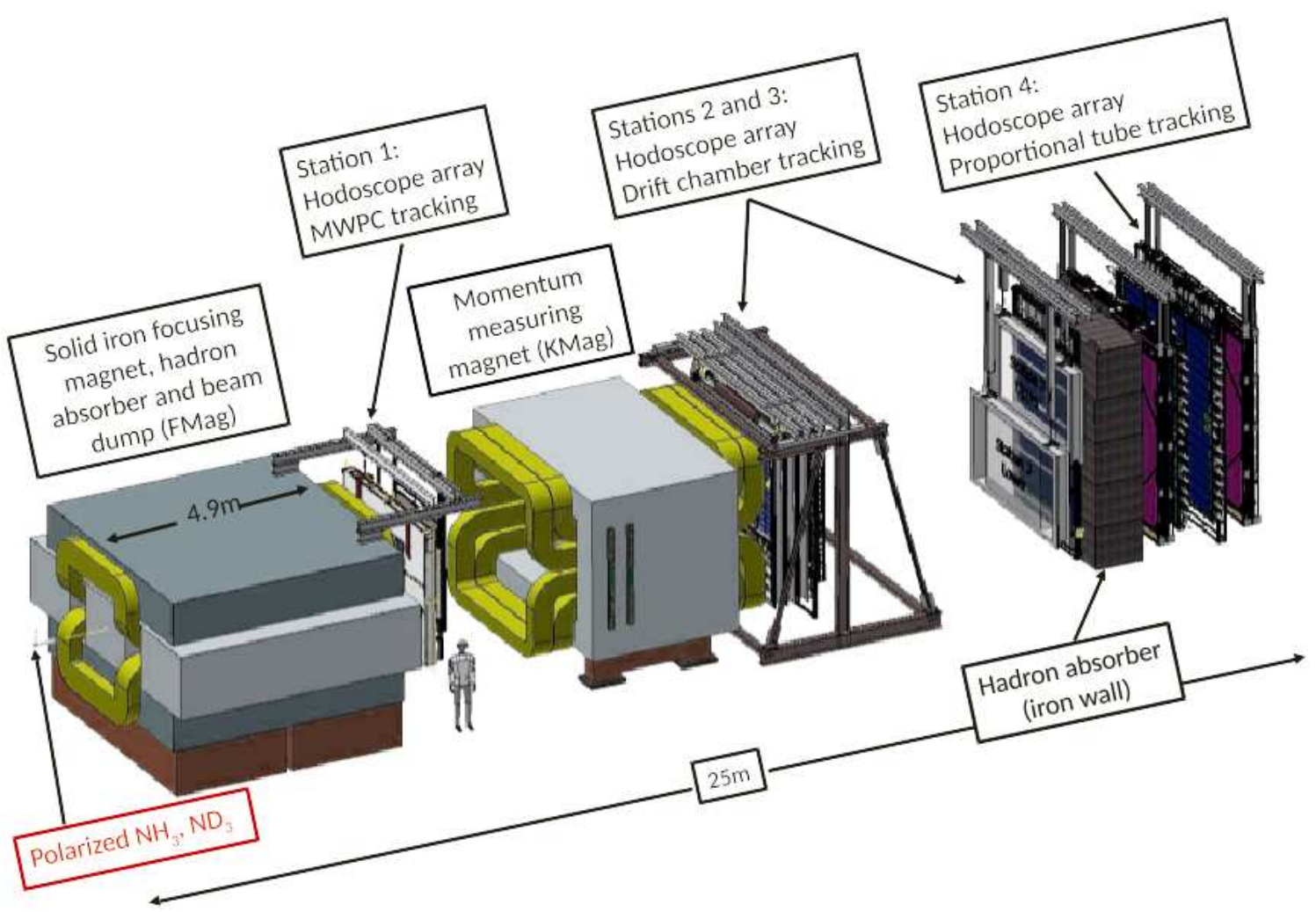

Figure 6: The spectrometer of the SeaQuest/E906 is used in SpinQuest experiment.

Shown in Figure $\rceil$ is the polarized target system of the SpinQuest. The target is polarized using Dynamic Nuclear Polarization as described in [35]. The frozen ammonia beads of $\mathrm{NH}_{3}$ and $\mathrm{ND}_{3}$ will be placed in the target holder in a $5 \mathrm{~T}$ magnet field. The whole container is cooled down to $1 \mathrm{~K}$ in a He evaporation refrigerator. In a $5 \mathrm{~T}$ field at $1 \mathrm{~K}$, electrons are nearly fully polarized. The electron polarization is then transferred to nucleon polarization through dipole-dipole interaction between the electron and the nucleon. By applying a suitable microwave signal, the desired spin state is populated. NMR detectors are placed near the targets to monitor the target polarization. An average polarization of $80 \%$ is expected for the $\mathrm{NH}_{3}$ target.

The preparation of the SpinQuest is well underway and first physics data-taking is expected to begin in September of 2019. Stay tuned.

\section{References}

[1] V. Barone, A. Drago. P. G. Ratcliffe, Phys. Rep. 359 (2002) 1, arXiv:hep-ph/0104283 


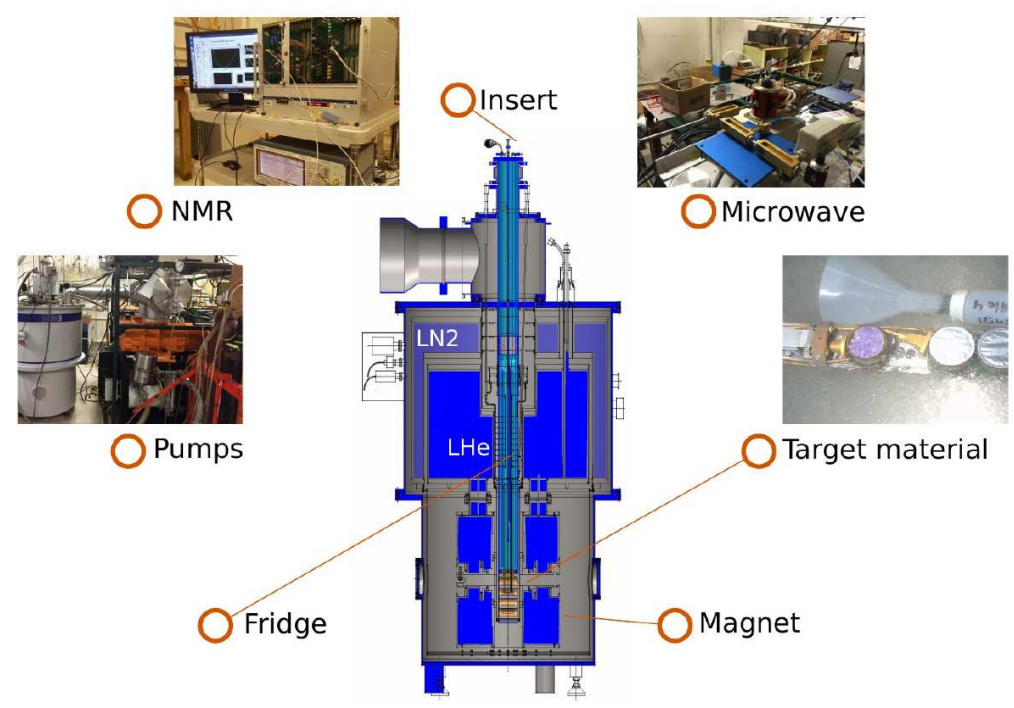

Figure 7: The target system of SpinQuest shown together with its supporting devices.

[2] S. J. Brodsky, D. S. Hwang, I. Schmidt, Phys. Lett. B 530 (2002) 99, arXiv:hep-ph/0201296; J. C. Collins, Phys. Lett. B536 (2002) 43, arXiv:hep-ph/0204004.

[3] X. Ji, Phys. Rev. Lett. 110 (2013) 262002, arXiv:1305.1539 [hep-ph].

[4] D. W. Sivers, Phys. Rev. D 41 (1990) 83.

[5] D. Boer and P. J. Mulders, Phys. Rev. D 57 (1998) 5780, arXiv:hep-ph/9711485.

[6] NA10 Collaboration, S. Falciano et al., Z. Phys. C 31 (1986) 513; M. Guanziroli et al., Z. Phys. C 37 (1988) 545.

[7] J. S. Conway et al., [E615 Collaboration], Phys. Rev. D 39 (1989) 92.

[8] L. Y. Zhu et al., [NuSea Collaboration], Phys. Rev. Lett. 99 (2007) 082301, arXiv:hep-ex/0609005.

[9] L. Y. Zhu et al., [NuSea Collaboration], Phys. Rev. Lett. 102 (2009) 182001, arXiv:0811.4589 [nucl-ex].

[10] L. Adamczyk et al., [STAR Collaboration], Phys. Rev. Lett. 116 (2016) 132301, arXiv:1511.06003 [nucl-ex].

[11] M. Aghasyan et al., [COMPASS Collaboration], Phys. Rev. Lett. 119 (2017) 112002, arXiv: 1704.00488 [hep-ex].

[12] A. Klein, X. Jiang, et al., Fermilab proposal E1039 (2013).

[13] G. Geesaman et al., Fermilab proposal E906 (1999).

[14] A. Airapetian et al., [HERMES Collaboration], Phys. Rev. Lett. 94 (2005) 012002, arXiv:hep-ex/0408013.

[15] A. Airapetian et al., [HERMES Collaboration], Phys. Rev. Lett. 103 (2009) 152002, arXiv:0906.3918 [hep-ex]. 
[16] V. Yu. Alexakhin et al., [COMPASS Collaboration], Phys. Rev. Lett. 94 (2005) 202002, arXiv:hep-ex/0503002.

[17] C. Adolph et al., [COMPASS Collaboration], Phys. Lett. B 744 (2015) 250, arXiv:1408.4405 [hep-ex].

[18] C. Adolph et al., [COMPASS Collaboration], Phys. Lett. B 770 (2017) 138, arXiv:1609.07374 [hep-ex].

[19] X. Qian et al., [JLab Hall A Collaboration], Phys. Rev. Lett. 107 (2011) 072003, arXiv:1106.0363 [nucl-ex].

[20] M. Anselmino et al., Eur. Phys. J. A 39 (2009) 89, arXiv:0805.2677 [hep-ph].

[21] P. Sun and F. Yuan, Phys. Rev. D 88 (2013) 034016, arXiv:1304.5037 [hep-ph].

[22] W. C, Cheng and J. C. Peng, Prog. Part. Nucl. Phys. 79 (2014) 95, arXiv:1406.1260 [hep-ph].

[23] E. A. Hawker et al., [NuSea Collaboration], Phys. Rev. Lett. 80 (1998) 3715, arXiv:hep-ex/9803011.

[24] M. Wakamatsu, Eur. Phys. J. A 44 (2010) 297, arXiv:0908.0972 [hep-ph].

[25] M. Deka et al., Phys. Rev. D 91 (2015) 014505.

[26] J. C. Collins, Nucl. Phys. B 396 (1993) 161, arXiv:hep-ph/9208213.

[27] A. Airapetian et al., [HERMES Collaboration], Phys. Lett. B 693 (2010) 11, arXiv:1006.4221 [hep-ex].

[28] E. S. Ageev et al., [COMPASS Collaboration], Nucl. Phys. B 765 (2007) 31, arXiv:hep-ex/0610068.

[29] C. Adolph et al., [COMPASS Collaboration], Phys. Lett. B 717 (2012) 376, arXiv:1205.5121 [hep-ex].

[30] M. Anselmino et al., Phy. Rev. D 87 (2013) 094019, arXiv:1303.3822 [hep-ph]

[31] K. Abe et al., [Belle Collaboration], Phys. Rev. Lett. 96 (2006) 232002, arXiv:hep-ex/0507063.

[32] A. Airapetian et al. (HERMES), PRL 95, 242001 (2005), arXiv:hep-ex/0506018.

[33] P. Hoodbhoy, R.L. Jaffe and A. Manohar, Nucl. Phys. B312 (1989) 571.

[34] S. Kumano and Qin-Tao Song, Phys Rev, D94, 054022 (2016), arXiv:1606.03149 [hep-ph].

S. Kumano, J. Phys. Conf. Ser. 543 (2014) 012001.

[35] D. Crabb, Nucl. Instr. Meth. A 356 (1995) 9. 